\title{
Impact of abnormal longitudinal rotation on the assessment of right ventricular systolic function in patients with severe pulmonary hypertension
}

\author{
Patrick Collier ${ }^{1}$, Bo Xu ${ }^{1}$, Kenya Kusunose ${ }^{1}$, Dermot Phelan ${ }^{1}$, Andrew Grant ${ }^{1}$, Paaladinesh \\ Thavendiranathan $^{1,2}$, Brian P. Griffin ${ }^{1}$, Richard A. Grimm ${ }^{1}$, Thomas H. Marwick ${ }^{1,3}$, Zoran B. Popović ${ }^{1}$ \\ ${ }^{1}$ Department of Cardiovascular Medicine, Cleveland Clinic, Cleveland, OH, USA; ${ }^{2}$ Department of Cardiology, University of Toronto, Toronto, \\ Ontario, Canada; ${ }^{3}$ The Baker Heart and Diabetes Institute, Melbourne, Australia \\ Contributions: (I) Conception and design: All authors; (II) Administrative support: B Xu, ZB Popović; (III) Provision of study materials or patients: \\ ZB Popović, P Collier, BP Griffin, RA Grimm; (IV) Collection and assembly of data: ZB Popović, P Collier, K Kusunose, A Grant, D Phelan; (V) \\ Data analysis and interpretation: ZB Popović, B Xu, P Collier, BP Griffin, RA Grimm; (VI) Manuscript writing: All authors; (VII) Final approval of \\ manuscript: All authors. \\ Correspondence to: Zoran B. Popović, MD, PhD. Heart and Vascular Institute, Department of Cardiovascular Medicine, Cleveland Clinic, 9500 Euclid \\ Avenue, Desk J1-5, Cleveland, OH 44195, USA. Email: popoviz@ccf.org.
}

Background: Assessment of right ventricular (RV) function plays an important role in patients with cardiopulmonary disease, and current guidelines recommend parameters including tricuspid annular plane systolic excursion (TAPSE) and right ventricular systolic excursion velocity (RVS') to assess RV longitudinal function. We assessed the hypothesis that the previously undescribed motion of RV longitudinal rotation (RVLR) is an independent predictor of both TAPSE and RVS'.

Methods: We assessed a series of 100 consecutive patients with pulmonary hypertension (PH) undergoing echocardiography. Patients with left ventricular (LV) dilation and dysfunction were excluded. Standard RV parameters were determined using established guidelines, while RVLR and right ventricular global longitudinal strain (RVGLS) measurements were performed using 2-dimensional (2D) speckle tracking technique.

Results: Mean peak RVLR measured $-4.2 \pm 3.7$ degrees. By convention, negative values implied clockwise motion. In a multiple linear regression model, TAPSE could be predicted from a combination of RVLR and RVGLS ( $\mathrm{R}=0.56, \mathrm{P}<0.001)$. A similar relationship was found for RVS' which could also be predicted from a combination of RVLR and RVGLS $(\mathrm{R}=0.52, \mathrm{P}<0.001)$. While no association was found between RVLR and RV size, estimated RV systolic pressure (RVSP) or the presence of a pericardial effusion, a mild correlation was noted between RVLR and QRS duration ( $\mathrm{R}=0.25, \mathrm{P}=0.01)$.

Conclusions: RVLR is an independent predictor of TAPSE and RVS'. Awareness of this motion should be considered in the interpretation of TAPSE and RVS' values as markers of RV systolic function, as abnormal RVLR may account for exaggerated values, particularly in patients with $\mathrm{PH}$ and RV dysfunction.

Keywords: Echocardiography; right ventricle; longitudinal rotation; strain imaging

Submitted Jan 12, 2018. Accepted for publication May 10, 2018.

doi: $10.21037 /$ jtd.2018.07.118

View this article at: http://dx.doi.org/10.21037/jtd.2018.07.118

\section{Introduction}

Right ventricular (RV) systolic function is an important determinant of prognosis in patients with pulmonary hypertension $(\mathrm{PH})(1,2)$. Indeed, current guidelines recommend that echocardiographic assessment of $\mathrm{RV}$ systolic function be carried out as an important component of the follow-up care of patients with PH (1). Given the 
well-documented complexity involved in echocardiographic assessment of RV systolic function (2), it is not surprising that a number of echocardiographic methods for evaluating $\mathrm{RV}$ systolic function have been used in clinical practice. Frequently used, recommended parameters include fractional area change (FAC), tricuspid annular plane systolic excursion (TAPSE), and pulsed wave tissue Doppler tricuspid annular systolic longitudinal excursion velocity (RVS') (3). Both TAPSE and RVS' assess RV longitudinal function (with the inference that the greater the descent or speed of descent of the base in systole, the better the RV systolic function), and when these parameters decreased, it has been shown to reflect RV dysfunction, and to be of prognostic value in $\mathrm{PH}(4-6)$. RV strain (the percentage change in myocardial deformation) has emerged as a novel marker of RV systolic function, serving as a marker of both regional and global RV contractility. It has the theoretical advantage of being able to distinguish between myocardial deformation and myocardial displacement (3).

Recently, in our echocardiographic laboratory, we observed the occurrence of an abnormal RV rocking motion in patients with $\mathrm{PH}$ that bore some similarities to our prior findings of abnormal left ventricular (LV) longitudinal rotation in the setting of $\mathrm{LV}$ dilatation and dysfunction (7-9). The aims of this study were to: (I) assess longitudinal rotation of the RV (RVLR) in patients with $\mathrm{PH}$; (II) determine whether such RVLR might have an impact on the conventional echocardiographic parameters used in the assessment of overall RV systolic function (i.e., TAPSE and RVS'); and (III) determine whether RVLR has an impact on prognosis.

\section{Methods}

\section{Study sample}

We studied patients in whom an echocardiogram was performed in an outpatient setting, as a part of the initial visit or subsequent follow-up evaluation, at the request of one of the PH specialists of the Cleveland Clinic Respiratory Institute. All consecutive patients seen during the period between April $1^{\text {st }} 2011$ and April $1^{\text {st }} 2012$, who fulfilled the inclusion criteria were included. The inclusion criteria were: an estimated systolic pulmonary arterial pressure of $>60 \mathrm{mmHg}$ on a formal echocardiogram report, and performance of the echocardiogram study using either Vivid 7 or Vivid 9 ultrasound systems (GE Medical, Milwaukee, USA). This was necessary, because only images acquired using these machines were amenable to the relevant analysis using the proprietary automated software (EchoPAC, Version 110.0.0. Advanced Analysis Technologies; GE Medical, Milwaukee, USA). Exclusion criteria were: PH due to left heart disease [i.e., Group 2 according to World Health Organization classification of PH (10)]; incomplete study (limited acquisition of RV and LV images); and suboptimal echocardiographic image quality precluding accurate measurement of $\mathrm{RV}$ and $\mathrm{LV}$ parameters (defined as inadequate visualization of more than two ventricular segments, or significant artifact).

The study protocol was approved by the Institutional Review Board (IRB) of the Cleveland Clinic Foundation (IRB number: 12-347). The medical records of all included patients were further reviewed to gather clinical data.

\section{Data analysis}

Transthoracic echocardiograms were reviewed and analyzed by two independent, experienced echocardiography readers. Standard echocardiographic measurements of the LV and RV were made in accordance with current guidelines $(3,11)$. Quantitative estimation of RV systolic function was carried out using FAC. Maximal systolic excursion of the tricuspid annulus in the longitudinal plane was measured using 2-dimensional (2D) images. RVS' was measured by tissue Doppler imaging from an apical 4-chamber window, with the pulsed Doppler sample volume placed at the tricuspid annulus. Measurement of right ventricular global longitudinal strain (RVGLS) was performed by speckletracking using EchoPAC (Version 110.0.0. Advanced Analysis Technologies; GE Medical, Chicago, Illinois, USA) as previously described (12). Estimated peak RV systolic pressures (RVSP) were calculated by summing the tricuspid gradient (estimated by the modified Bernoulli equation from the tricuspid regurgitant velocity) and the right atrial pressure (estimated by inferior vena cava size and changes with respiration). RVLR was calculated by off-line analysis using EchoPAC (Version 110.0.0. Advanced Analysis Technologies; GE Medical, Chicago, Illinois, USA). In brief, following selection of the optimal quality $2 \mathrm{D}$ digital images in a dedicated RV long-axis view, the endocardium was traced in an optimal frame, from which a speckle tracking region of interest was automatically selected to approximate the myocardium between the endocardium and epicardium. The region of interest width was adjusted as needed to fit the wall thickness. Measurements were deemed unacceptable, if two or more segments did not track 
adequately after two manual adjustments of the endocardial borders. The software then automatically selected suitable, stable objects for tracking and searched for them in the next frame using the sum of absolute differences algorithm before computing an RVLR profile defining the ventricular centroid from the mid-myocardial line on each frame. Peak systolic RVLR was defined as the maximal rotation value of the RV during systole. In accordance with engineering notation, the negative sign indicated clockwise rotation, and a positive sign indicated counterclockwise rotation.

\section{In vitro studies}

Because speckle tracking rotation quantitation software was designed to measure angular motion of rotationally symmetrical object occurring relative to the point of reference that coincides with its center of gravity, we performed an in vitro experiment.

Commercially obtained porcine hearts weighing between 200 and 250 grams were transected in order to obtain a slice ( $15 \mathrm{~mm}$ in thickness) corresponding to the apical fourchamber view. The hearts were then mounted on a circular plate that rotated around the axle passing either through the point corresponding to the base of the left ventricle, or to the point corresponding to the base of the right ventricle, and placed into a water tank. The plate was connected via a system of opposing springs to the rotating spindle that caused the motion of the plate in a see-saw manner, thus imitating the "rocking" motion of the heart. The diameter of the circle of rotating spindle was set to zero, maximum, and midway between zero and maximum. A total of 6 different setups were run, each of them imaged with three different frame rates (at 30 frames per second, 46 frames per second, and 57 frames per second). At each setup, two dimensional, $M$ mode, and pulsed wave tissue Doppler data were collected using a Vivid 9 echocardiographic machine (GE Medical, Milwaukee, USA) with a standard transthoracic probe held by a holder in a water tank.

For the gold standard of angular displacement, we analyzed the fiducial points on images obtained by the video camera (30 frames per second) placed over the water tank. Data were digitized using the Engauge Digitizer v.15 (Mark Mitchell, 2012; https://markummitchell.github.io/engaugedigitizer) with the change of plate angle used for reference. Correlations were assessed by Pearson correlation coefficient.

The in vitro studies confirmed the accuracy of RV rotation measurement with good agreement between the gold standard (in vitro experiment) and echocardiographyderived rotation, with an overall correlation coefficient $r$ of $0.98(\mathrm{P}<0.01)$.

\section{Statistical analysis}

Data were presented as either mean \pm standard deviations (SDs) or median [interquartile range (IQR)] for normally and non-normally distributed continuous variables, respectively, whereas frequencies and percentages (in parentheses) summarized categorical variables. Multiple linear regression analyses were performed to assess whether the dependent variables, TAPSE and RVS' could be predicted from a linear combination of the independent variables, RVLR and RVGLS. To assess inter-observer variability, RVLR was measured by two independent blinded investigators in a subgroup of 15 consecutive study patients using the Bland-Altman analysis. Intraobserver variability was measured by repeated assessment of RVLR in the same group of patients by one investigator at a separate time using Bland-Altman analysis. A Cox regression proportional hazards model was used to assess survival related to baseline covariates of TAPSE, RVGLS and RVLR. To control for the known impact of clinical parameters on prognosis, we first calculated the REVEAL score (13) of individual patients. Then, we performed nonparsimonious modelling of proportional hazards by first applying the REVEAL score and PH type (with patients dichotomized according to the presence or absence of type $1 \mathrm{PH})$ as predictors. In the second step, we performed forward stepwise regression using TAPSE, RVGLS, RVS' and RVLR as additional prognostic variables. Because crosscorrelation between TAPSE, RVGLS, RVS' and RVLR was expected, we attempted separate stepwise regression for each of these four parameters into the model.

Statistical analysis was performed using the statistical software packages, Sigma Plot (Version 11.0, Systat Software, Inc., San Jose, USA) and SPSS Statistics for Windows (Version 21.0., IBM Corp, Armonk, NY, USA). A $P$ value of $<0.05$ was considered significant.

\section{Results}

We identified 117 patients who fulfilled the clinical inclusion criteria. Out of this group, 11 patients were excluded due to inadequate image quality and a further 6 patients were excluded due to incomplete studies. Therefore, a total of 100 consecutively identified 
Table 1 Demographics and medication profiles of the study cohort

\begin{tabular}{lc}
\hline Parameter & Study cohort $(\mathrm{n}=100)$ \\
\hline Age, year & $58 \pm 15$ \\
Gender, male & $34(34 \%)$ \\
$\mathrm{BMI}, \mathrm{kg} / \mathrm{m}^{2}$ & $28[24-33]$ \\
$\mathrm{BSA}, \mathrm{m}^{2}$ & $1.9[1.7-2.1]$ \\
$\mathrm{SBP} / \mathrm{DBP}, \mathrm{mmHg}$ & $122 / 70 \pm 18 / 10$ \\
HR, bpm & $79 \pm 15$ \\
PR interval, ms & $168[152-183]$ \\
QRS duration, ms & $98[86-107]$ \\
PDE5 inhibitor therapy & $65(65 \%)$ \\
Prostacyclin therapy & $46(46 \%)$ \\
Endothelin receptor antagonist & $34(34 \%)$ \\
\hline
\end{tabular}

Values stand for mean \pm SDs, median [IQR] or $n(\%)$. BMI, body mass index; BSA, body surface area; SBP/DBP, systolic and diastolic blood pressure; HR, heart rate. patients formed the final study population. The mean patient age was: $58 \pm 15$ years. Baseline demographics and medication use are summarized in Table 1. According to the classification of $\mathrm{PH}$ based on current guidelines, the etiologies of $\mathrm{PH}$ in the study population were as follows: Group 1-pulmonary arterial hypertension (80\%), Group 2-PH due to left heart disease (0\%), Group 3-PH due to lung disease and/or hypoxia (5\%), Group 4-chronic thromboembolic PH (10\%), and Group 5-PH with unclear and/or multifactorial mechanisms (5\%) (1). For 88 of the 100 study patients $(88 \%)$, invasive hemodynamic data were available, with a mean right atrial pressure of $12 \pm 6 \mathrm{mmHg}$, a mean pulmonary artery pressure of $55 \pm 18 \mathrm{mmHg}$, a mean wedge pressure of $12 \pm 7 \mathrm{mmHg}$, and a mean cardiac index (Fick method) of $2.4 \pm 0.9 \mathrm{~L} / \mathrm{min} / \mathrm{m}^{2}$.

The tracking of rotational motion of the right ventricle in a set of systolic images obtained in apical 4-chamber views obtained in a patient with a prominent clockwise RVLR is demonstrated in Figure 1, while the video clip obtained

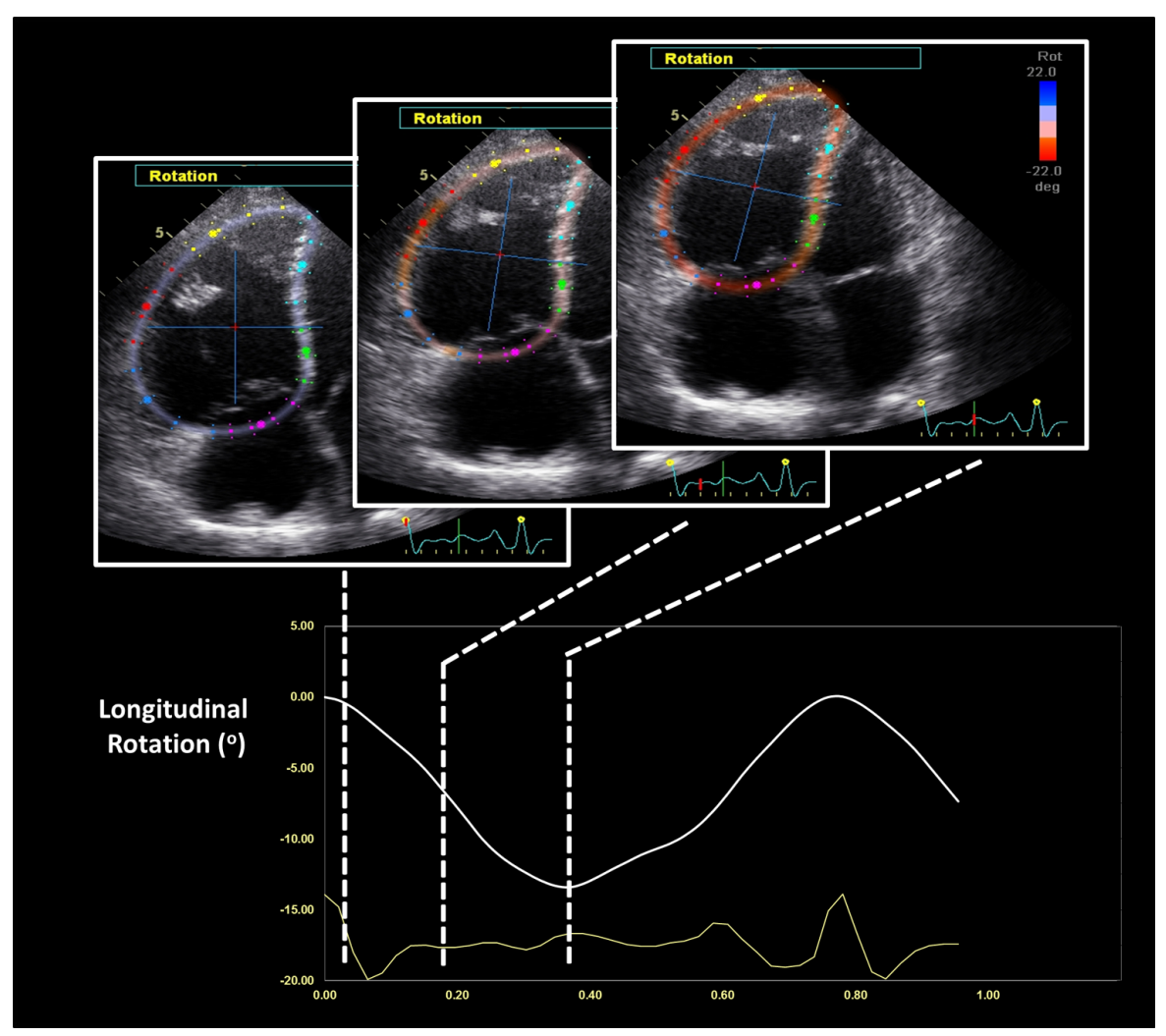

Figure 1 RVLR measurement technique. Annotated measurements of RVLR and derivation of peak rotation from a rotation curve. Video files of these images are available as supplementary files (Figures S1,S2: long-axis RV echocardiographic clips demonstrating measurement of abnormal RV clockwise rotation in a patient with severe pulmonary hypertension with and without annotation). RVLR, right ventricular longitudinal rotation. 
Table 2 Echocardiographic data of the study cohort

\begin{tabular}{lc}
\hline Parameter & Study cohort $(\mathrm{n}=100)$ \\
\hline LAVI, mL & $26[24-37]$ \\
LVEDV, $\mathrm{mL}$ & $66[47-92]$ \\
LVESV, $\mathrm{mL}$ & $23[16-33]$ \\
LVEF, \% & $64 \pm 7$ \\
RVSP, mmHg & $87[74-99]$ \\
RAP, mmHg & $5[5-10]$ \\
RVEDA, cm ${ }^{2}$ & $32[25-43]$ \\
RV FAC, \% & $27.3 \pm 8.9$ \\
Peak RVGLS, \% & $-13.9 \pm 4.6$ \\
TAPSE, mm & $17.6 \pm 4.4$ \\
RVS', cm/s & $11.2 \pm 3.1$ \\
Peak RVLR, deg & $-4.2 \pm 3.7$ \\
Any pericardial effusion, $\mathrm{n}$ & $37(37 \%)$ \\
\hline
\end{tabular}

Values stand for mean \pm SDs, median [IQR] or $n(\%)$. LAVI, left atrial volume index; LVEDV, left ventricular end-diastolic volume; LVESV, left ventricular end-systolic volume; LVEF, left ventricular ejection fraction; RVSP, right ventricular systolic pressure; RAP, right atrial pressure; RVEDA, right ventricular end-diastolic area; FAC, fractional area change; RVGLS, right ventricular global longitudinal strain; TAPSE, tricuspid annular plane systolic excursion; RVS', right ventricular systolic excursion velocity; RVLR, right ventricular longitudinal rotation.

in the same patient is available as Figures $S 1$ and $S 2$. All echocardiographic variables are summarized in Table 2. In keeping with inclusion criteria of severe $\mathrm{PH}$, right heart catheterization data revealed a mean pulmonary artery pressure of $55 \pm 18 \mathrm{mmHg}$, while median (IQR) estimated RVSP measured 87 [74-99] mmHg. Most patients had RV enlargement with median RV end-diastolic area of 32 [25-43] $\mathrm{cm}^{2}$ [compared to lower reference value (LRV) $\pm 95 \%$ confidence intervals (CIs) of $25 \%$ (24-27\%) as per guidelines] (3). This study population also had documented RV systolic dysfunction with decreased mean FAC values of $27.3 \% \pm 8.9 \%$ [LRV $\pm 95 \%$ CIs of $35 \%(32-38 \%)$ as per guidelines] (3). Consistent with this finding, RV deformation was also abnormally decreased, with mean RVGLS values of $-13.9 \% \pm 4.6 \%$ [LRV $\pm 95 \%$ CIs of $-20 \%(-15 \%$ to $-24 \%)$ ], which was approximately half of published normal values (3). In contrast, TAPSE and RVS' were relatively preserved, with mean TAPSE values of $17.6 \pm 4.4 \mathrm{~mm}$ \{compared to $\mathrm{LRV} \pm 95 \%$ CIs of
16 [15-18] $\mathrm{mm}$ \} and mean RVS' values of $11.2 \pm 3.1 \mathrm{~cm} / \mathrm{s}$ [compared to LRV $\pm 95 \%$ CIs of $6 \mathrm{~mm}(5-7 \mathrm{~mm})$ ] (3). While 37 patients had identifiable pericardial effusions, the majority of these pericardial effusions were either trivial $(\mathrm{n}=16)$ or small $(\mathrm{n}=17)$. Peak RVLR measured $-4.2^{\circ} \pm 3.7^{\circ}$. Absolute inter-observer variability was $-0.6^{\circ} \pm 1.2^{\circ}(95 \%$ CIs of mean of differences: $-1.3^{\circ}$ to $\left.0.1^{\circ}\right)$. The error was random with no systematic trend or proportional bias observed $(\mathrm{P}=0.36)$, while the inter-observer coefficient of repeatability was $2.4^{\circ}$. Absolute intra-observer variability was $-0.1^{\circ} \pm 1.0^{\circ}\left(95 \%\right.$ CIs of mean of differences: $-0.4^{\circ}$ to $0.6^{\circ}$ ). Again, the error was random with no systematic trend or proportional bias observed $(\mathrm{P}=0.19)$, while the intraobserver coefficient of repeatability was $2.0^{\circ}$.

Multiple linear regression demonstrated that TAPSE can be predicted from a linear combination of peak RVLR and peak RVGLS as follows: TAPSE $=10.3-(0.3 \times$ RVLR $)-$ $(0.4 \times$ RVGLS); $\mathrm{R}=0.56(\mathrm{P}<0.001)$ (Figure $2 A, B)$. Similarly, RVS' can be predicted from a linear combination of peak RVLR and peak RVGLS as follows: RVS' $=7.1-$ $(0.3 \times \mathrm{RVLR})-(0.2 \times \mathrm{RVGLS}) ; \mathrm{R}=0.52(\mathrm{P}<0.001)$ (Figure $3 A, B)$. While no association was found between RVLR and RV size, estimated RVSP or the presence of a pericardial effusion, a mild correlation was noted between longitudinal $\mathrm{RV}$ rotation and $\mathrm{QRS}$ duration $(\mathrm{R}=0.25$, $\mathrm{P}=0.01)$.

Over a median follow-up period of 996 [533-1,098] days, 41 patients died. In univariate analysis, RVGLS, TAPSE and RVLR correlated with patient survival, while RVS' showed trends towards significance. In a multivariate analysis, only RVGLS had significant incremental prognostic value $(\mathrm{P}=0.048)$ over and above the REVEAL score, and the etiology of $\mathrm{PH}$ (Table 3).

\section{Discussion}

This study investigated a previously undescribed movement of the RV in the setting of severe $\mathrm{PH}$, namely clockwise longitudinal rotation. We demonstrated that such rotatory (or rocking) motion influenced both TAPSE and RVS', both of which are important conventional measures of RV systolic function, and markers of prognosis (4-6). In particular, greater degrees of clockwise rotation were associated with greater amounts of both tricuspid annular displacements (as measured by TAPSE) and systolic velocity (RVS'). However, the values of these parameters may be falsely reassuring despite the presence of significant RV dysfunction. 
A

Multiple linear regression model

TAPSE $(\mathrm{mm})=10.3-0.3 \times \mathrm{RV}$ rotation $(\mathrm{deg})-0.4 \mathrm{RV}$ strain (\%)

$R=0.56, P<0.001, n=100$
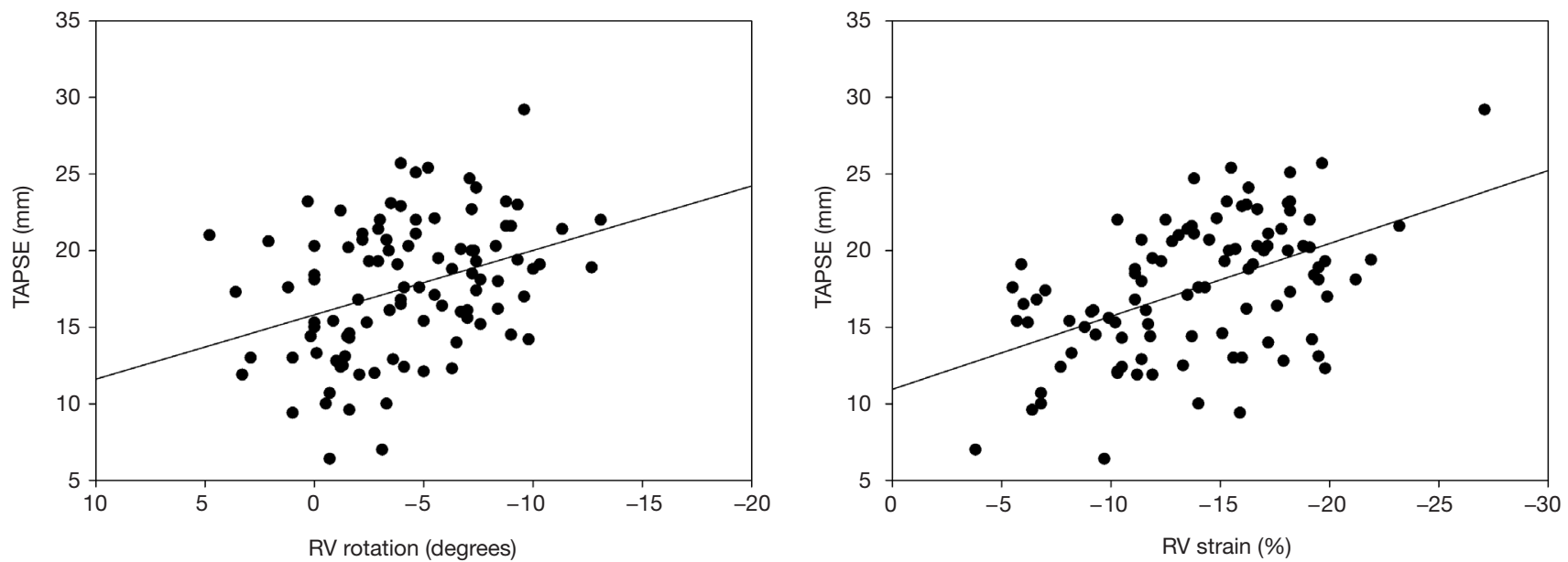

B

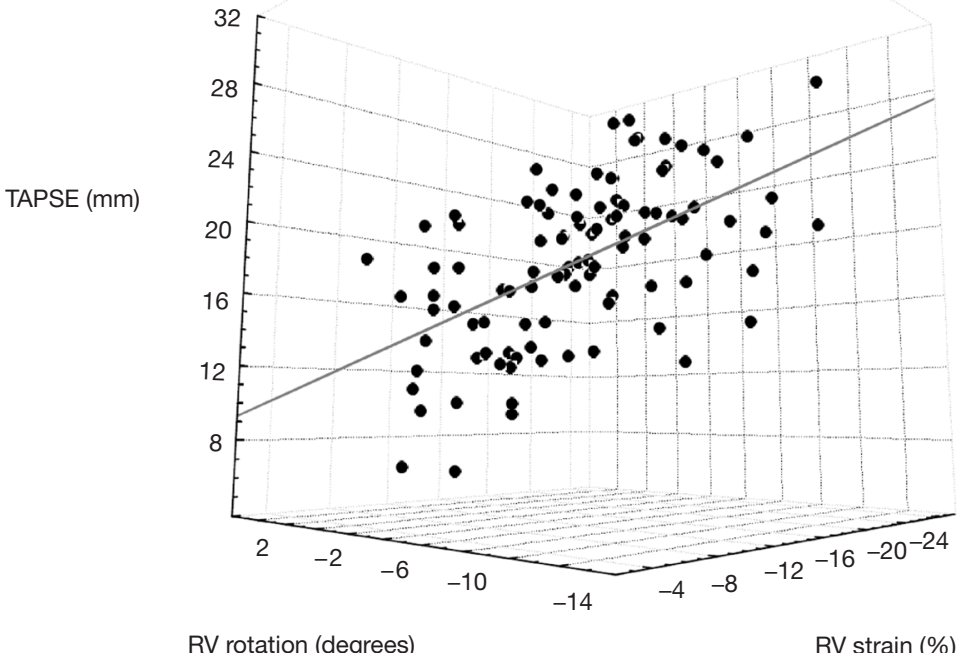

Figure 2 Multiple regression model for TAPSE using RVLR and RVGLS. Peak RVLR and RVGLS were found to be predictive of TAPSE, and the results are shown in 2D regression (A) and three-dimensional regression curves (B). TAPSE, tricuspid annulus peak systolic excursion; RVLR, right ventricular longitudinal rotation; RVGLS, right ventricular global longitudinal strain.

Prior studies that investigated the different parameters of RV systolic function have shown that FAC, TAPSE and RVS' were generally concordant (14). In contrast, in the present cohort of consecutive patients with severe $\mathrm{PH}$, we found FAC and TAPSE to be somewhat discordant, i.e., FAC was markedly reduced, while TAPSE and RVS' levels were found to be above the published LRV (even if still below the means) (3). Consideration of these findings with reference to the three discrete elements known to incorporate RV systolic function may however offer further insight. Contraction of spiral longitudinal muscles (which produces systolic basal-apical shortening), inter-ventricular 
A

Multiple linear regression model

$\mathrm{RV}$ systolic annular velocity $(\mathrm{cm} / \mathrm{s})=7.1-0.3 \times \mathrm{RV}$ rotation $(\mathrm{deg})-0.2 \mathrm{RV}$ strain $(\%)$

$R=0.52, P<0.001, n=100$
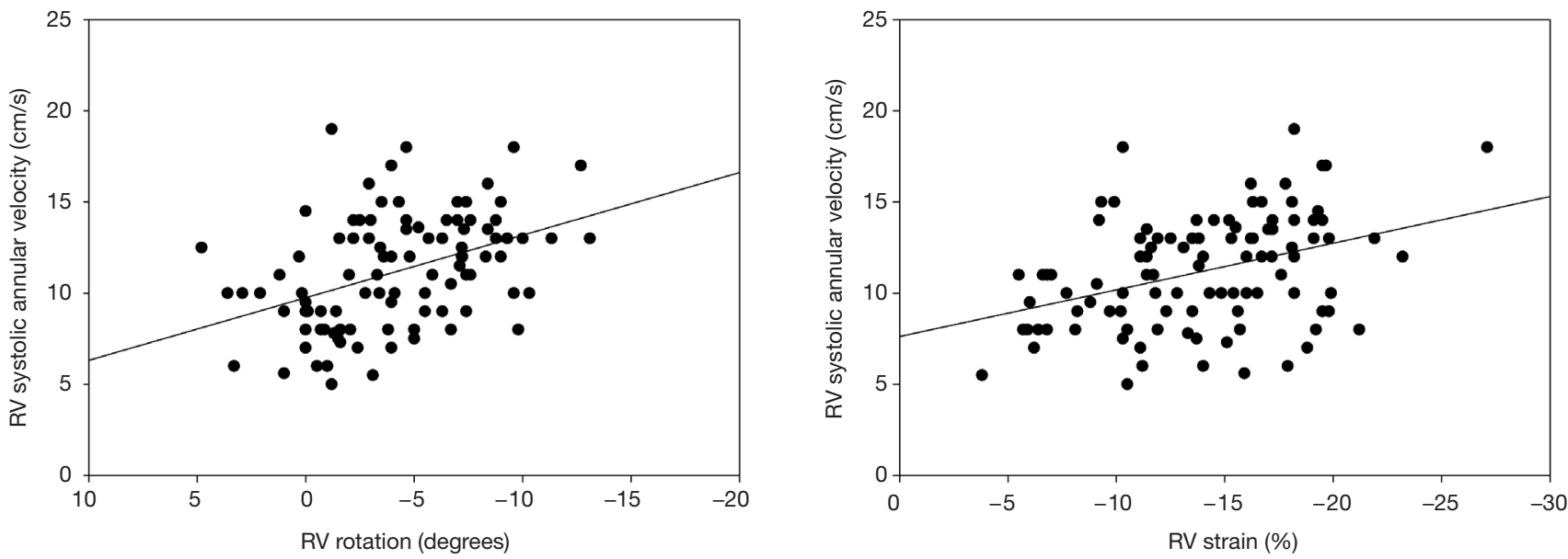

B

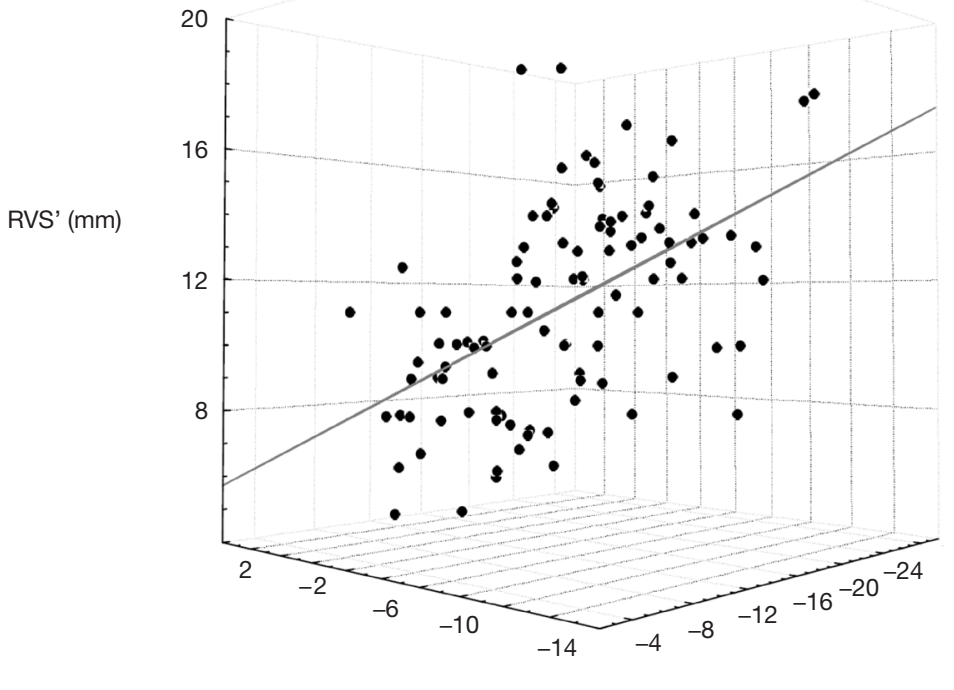

$\mathrm{RV}$ rotation (degrees)

RV strain (\%)

Figure 3 Multiple regression model for RVS' using RVLR and RVGLS. Peak RVLR and RVGLS were found to be predictive of RVS', and the results are shown in 2D regression (A) and three-dimensional regression curves (B). RVS', right ventricular peak systolic velocity; RVLR, right ventricular longitudinal rotation; RVGLS, right ventricular global longitudinal strain.

septal motion (affected by biventricular interdependence and conduction (and motion of the RV free wall are important determinants of FAC, whereas longitudinal function alone is the predominant determinant of TAPSE and RVS'.

Peak RVLR and RVGLS were found to be predictive of both TAPSE and RVS', although regression coefficients $(\mathrm{R}=0.56$ and 0.52 , respectively) were relatively modest. However, RV remodeling in $\mathrm{PH}$ is clearly a complex phenomenon involving a structure with an already complex geometry. Indeed, a multitude of modifying factors may exist, not the least of which includes differences in afterload, 
Table 3 Results from univariate and multivariate Cox proportional hazards models

\begin{tabular}{lccccc}
\hline Statistical model & $\mathrm{B}$ & $\mathrm{SE}$ & Wald & Sig. & $95 \%$ Cls for HR \\
\hline Univariate model & & & & & \\
RVGLS & 0.12 & 0.04 & 10.90 & 0.001 & $1.08-1.16$ \\
TAPSE & -0.11 & 0.04 & 8.81 & 0.003 & $0.87-0.93$ \\
RVLR & 0.09 & 0.04 & 4.94 & 0.026 & $1.05-1.15$ \\
RVS' & -0.09 & 0.05 & 2.68 & 0.102 & $0.87-0.97$ \\
Multivariate model & & & & 0.000 & $1.25-1.69$ \\
REVEAL score & 0.38 & 0.08 & 24.44 & 0.033 & $1.07-4.56$ \\
PH Group 1 & 0.79 & 0.37 & 4.54 & 0.049 & $1.00-1.15$ \\
RVGLS & 0.07 & 0.04 & 3.86 & & \\
\hline
\end{tabular}

$\mathrm{PH}$, pulmonary hypertension; RVGLS, right ventricular global longitudinal strain; RVLR, right ventricular longitudinal rotation; RVS', right ventricular systolic excursion velocity; TAPSE, tricuspid annular plane systolic excursion

ventricular hypertrophy, fibrosis, ischemia, wall stress and the degree of flattening of the inter-ventricular septum. One such factor that may amplify the effects of RV rotation on tricuspid annular motion is the degree of RV dilation. This is known to occur relatively early, in response to increased afterload on the right side of the heart compared to the left, due to the relatively increased compliance and lower mass/ volume ratio of the RV (15-17). However, we did not find a relationship between peak RVLR and RV size, which may relate to the fact that most patients already had significant RV dilation. Although pericardial effusions are commonly found in association with $\mathrm{PH}$, our data suggested that the absence or presence of a pericardial effusion appears to have little or no role in augmenting rotatory motion, and this may relate to our previous findings that only a relatively small amount of energy is actually required to induce longitudinal rotation (7). Unique to the $\mathrm{RV}$ is the premier contribution played by longitudinal fibers to overall RV systolic function (15). It is not surprising therefore that, among non-volumetric echocardiographic indices, speckle tracking longitudinal RV strain was shown to exhibit the closest association with RV ejection fraction as determined by cardiac magnetic resonance imaging (the reference standard of RV systolic function) (18). Thus, a noteworthy finding in the current study is that the peak RVLR was of similar importance to speckle tracking longitudinal RV strain, in terms of the impact on TAPSE and RVS'.

The etiology of abnormal rotatory motion of the RV in the setting of $\mathrm{PH}$ without intrinsic $\mathrm{LV}$ dysfunction can only be speculated. One potential mechanism for abnormal rotation may relate to an imbalance of ventricular strains. We have previously shown that in patients with LV dysfunction, clockwise LV longitudinal rotation occurred in patients with high strains of lateral $\mathrm{LV}$ wall $(7,8)$. Furthermore, we have previously shown that in $\mathrm{PH}$, longitudinal LV lateral wall strain was typically preserved, while RV free wall and inter-ventricular septal longitudinal strains were typically reduced (proportional to the degree of pressure overload as assessed by either estimated RVSP or by the LV eccentricity index-a measure of septal displacement or flattening) (19). Thus, the presence of a ventricular strain gradient between the $\mathrm{LV}$ lateral wall (with preserved strain) and RV free wall may contribute to systolic clockwise rotation of the dilated, pressure-overloaded RV.

Although overall RV systolic function should never be assessed by annular motion alone, from a clinical practice point of view, TAPSE is a parameter that is more commonly obtained than other markers of RV systolic function, a point that is highlighted in the current echocardiographic guidelines (3). A likely explanation is that the RV free wall cannot be completely visualized, yet annular motion may still be clearly evident. In this scenario, however, increased clockwise rotation can still be qualitatively identified by assessing the relative angulation between the annular plane and the inter-ventricular septum. When RV contraction is preserved, this angle normally decreases. Our findings suggest that when this angle remains fixed, it could be speculated that "relative preservation" of annular motion may result from RVLR, rather than preserved RV contraction, raising the suspicion for possible significant 
Tricuspid annular planar systolic excursion/velocity

RV longitudinal

deformation

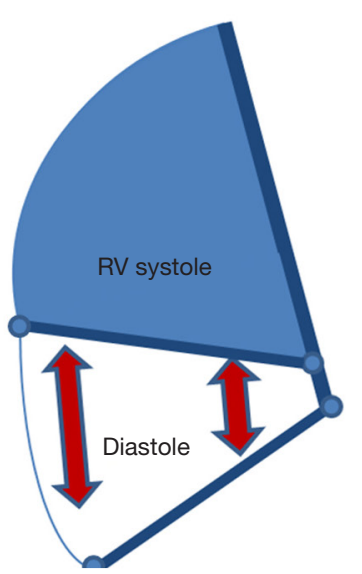

$+\quad$ RV longitudinal

rotation

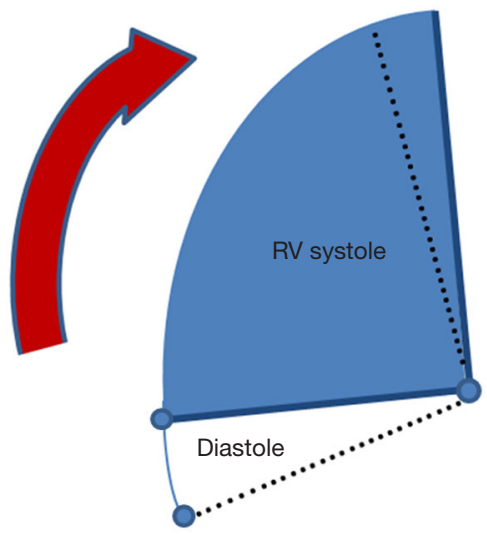

Figure 4 Normal versus increased clockwise rotation of the right ventricle can be qualitatively identified by assessing the relative angulation between the tricuspid annular plane and the inter-ventricular septum during systole.
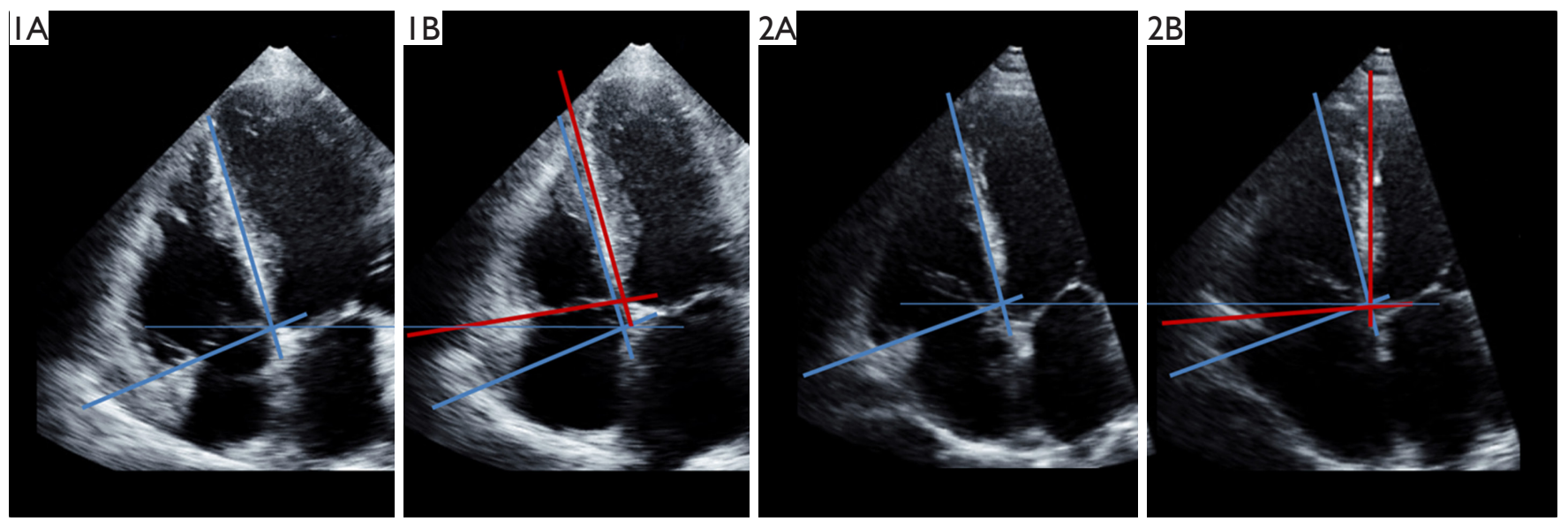

Figure 5 End-diastolic and end-systolic frames in a normal heart (two panels on the left) versus end-diastolic and end-systolic frames in a patient with pulmonary hypertension and increased clockwise rotation (two panels on the right).

underlying RV dysfunction (Figures 4,5). Awareness of the potential confounding contribution of RVLR to the "relative preservation" of annular motion may therefore limit the possibility of missing RV dysfunction, particularly where such a finding may have prognostic importance.

\section{Limitations}

Although this study population represented a consecutive series of patients, subjects were retrospectively identified and recruited into this study. Many of these patients with $\mathrm{PH}$ 
already had advanced RV systolic dysfunction. However, we would argue that the demonstration of relative preservation of TAPSE and RVS' in this context further emphasized our conclusions regarding the potential confounding contribution of RVLR. Additionally, the software used was not designed to specifically assess change in $\mathrm{RV}$ orientation in a horizontal plane. Possibly, better software, perhaps by separating lateral and septal walls, would have better accuracy to quantitate rotational motion of RV silhouette.

Further, the adjustment for clinical factors in the survival analysis was performed using the REVEAL score (13), which was developed specifically for the initial assessment of survival in type $1 \mathrm{PH}$. On the other hand, almost all of the parameters incorporated into the REVEAL score (e.g., functional class, age, kidney function, and blood pressure) were prognostic in other types of $\mathrm{PH}$. A larger dataset is needed to adequately assess the hazard associated with excessive RVLR. Nevertheless, our survival analysis was hypothesis-generating, and may serve as the rationale for a future evaluation of RVLR as a potential survival predictor for patients with $\mathrm{PH}$.

\section{Conclusions}

Clockwise angular displacement of the right ventricle (RVLR) was observed in a population of patients with $\mathrm{PH}$ and RV systolic dysfunction. RVLR influenced the measurements of the TAPSE and RVS' in these patients. In contrast, RVLR did not affect RV global longitudinal strain, which may indicate its potential usefulness as a prognostic marker in $\mathrm{PH}$.

\section{Acknowledgements}

None.

\section{Footnote}

Conflicts of Interest: The authors have no conflicts of interest to declare.

Ethical Statement: The study protocol was approved by the Institutional Review Board (IRB) of the Cleveland Clinic Foundation (IRB number: 12-347).

\section{References}

1. Galie N, Hoeper MM, Humbert M, et al. Guidelines for the diagnosis and treatment of pulmonary hypertension. Eur Respir J 2009;34:1219-63.

2. Jiang L, Levine RA, Weyman AE. Echocardiographic Assessment of Right Ventricular Volume and Function. Echocardiography 1997;14:189-206.

3. Rudski LG, Lai WW, Afilalo J, et al. Guidelines for the echocardiographic assessment of the right heart in adults: a report from the American Society of Echocardiography endorsed by the European Association of Echocardiography, a registered branch of the European Society of Cardiology, and the Canadian Society of Echocardiography. J Am Soc Echocardiogr 2010;23:685713; quiz 86-8.

4. Forfia PR, Fisher MR, Mathai SC, et al. Tricuspid annular displacement predicts survival in pulmonary hypertension. Am J Respir Crit Care Med 2006;174:1034-41.

5. Lindqvist $\mathrm{P}$, Waldenstrom $\mathrm{A}$, Henein $\mathrm{M}$, et al. Regional and global right ventricular function in healthy individuals aged 20-90 years: a pulsed Doppler tissue imaging study: Umea General Population Heart Study. Echocardiography 2005;22:305-14.

6. Mathai SC, Sibley CT, Forfia PR, et al. Tricuspid annular plane systolic excursion is a robust outcome measure in systemic sclerosis-associated pulmonary arterial hypertension. J Rheumatol 2011;38:2410-8.

7. Popovic ZB, Grimm RA, Ahmad A, et al. Longitudinal rotation: an unrecognised motion pattern in patients with dilated cardiomyopathy. Heart 2008;94:e11.

8. Phillips KP, Popovic ZB, Lim P, et al. Opposing wall mechanics are significantly influenced by longitudinal cardiac rotation in the assessment of ventricular dyssynchrony. JACC Cardiovasc Imaging 2009;2:379-86.

9. Stankovic I, Aarones M, Smith HJ, et al. Dynamic relationship of left-ventricular dyssynchrony and contractile reserve in patients undergoing cardiac resynchronization therapy. Eur Heart J 2014;35:48-55.

10. Simonneau G, Gatzoulis MA, Adatia I, et al. Updated clinical classification of pulmonary hypertension. J Am Coll Cardiol 2013;62:D34-41.

11. Lang RM, Bierig M, Devereux RB, et al. Recommendations for chamber quantification: a report from the American Society of Echocardiography's Guidelines and Standards Committee and the Chamber Quantification Writing Group, developed in conjunction with the European Association of Echocardiography, a branch of the European Society of Cardiology. J Am Soc Echocardiogr 2005;18:1440-63.

12. Chow PC, Liang XC, Cheung EW, et al. New two- 
dimensional global longitudinal strain and strain rate imaging for assessment of systemic right ventricular function. Heart 2008;94:855-9.

13. Benza RL, Gomberg-Maitland M, Miller DP, et al. The REVEAL Registry risk score calculator in patients newly diagnosed with pulmonary arterial hypertension. Chest 2012;141:354-62.

14. Tamborini G, Pepi M, Galli CA, et al. Feasibility and accuracy of a routine echocardiographic assessment of right ventricular function. Int J Cardiol 2007;115:86-9.

15. Haddad F, Hunt SA, Rosenthal DN, et al. Right ventricular function in cardiovascular disease, part I: Anatomy, physiology, aging, and functional assessment of the right ventricle. Circulation 2008;117:1436-48.

Cite this article as: Collier $\mathrm{P}, \mathrm{Xu} \mathrm{B}$, Kusunose $\mathrm{K}$, Phelan D, Grant A, Thavendiranathan P, Griffin BP, Grimm RA, Marwick TH, Popović ZB. Impact of abnormal longitudinal rotation on the assessment of right ventricular systolic function in patients with severe pulmonary hypertension. J Thorac Dis 2018;10(8):4694-4704. doi: 10.21037/jtd.2018.07.118
16. Chin KM, Rubin LJ. Pulmonary arterial hypertension. J Am Coll Cardiol 2008;51:1527-38.

17. Haddad F, Doyle R, Murphy DJ, et al. Right ventricular function in cardiovascular disease, part II: pathophysiology, clinical importance, and management of right ventricular failure. Circulation 2008;117:1717-31.

18. Leong DP, Grover S, Molaee P, et al. Nonvolumetric echocardiographic indices of right ventricular systolic function: validation with cardiovascular magnetic resonance and relationship with functional capacity. Echocardiography 2012;29:455-63.

19. Puwanant S, Park M, Popovic ZB, et al. Ventricular geometry, strain, and rotational mechanics in pulmonary hypertension. Circulation 2010;121:259-66. 


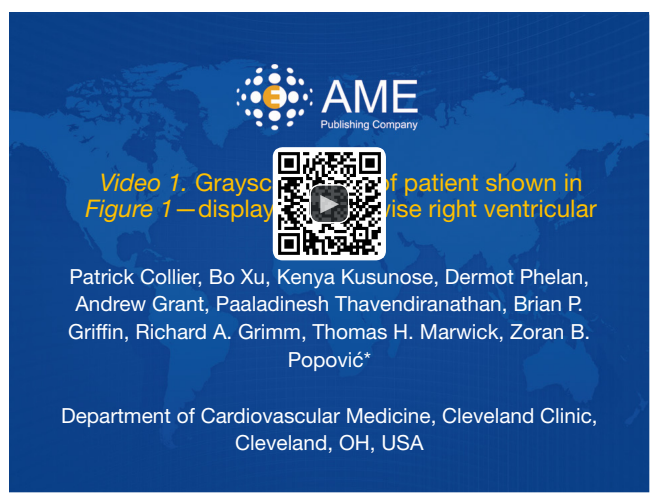

Figure S1 Grayscale video of patient shown in Figure 1-displaying clockwise right ventricular rotation (20). Available online: http://www. asvide.com/article/view/26622

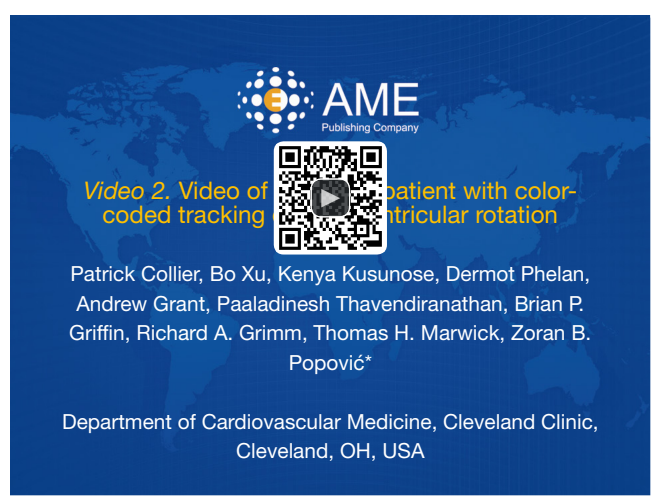

Figure S2 Video of the same patient with color-coded tracking of right ventricular rotation (21). Available online: http://www.asvide.com/ article/view/26623

\section{References}

20. Collier P, Xu B, Kusunose K, et al. Grayscale video of patient shown in Figure 1-displaying clockwise right ventricular rotation. Asvide 2018;5:706. Available online: http://www.asvide.com/article/view/26622

21. Collier P, Xu B, Kusunose K, et al. Video of the same patient with color-coded tracking of right ventricular rotation. Asvide 2018;5:707. Available online: http://www.asvide.com/article/view/26623 